\title{
Development of a new method for collecting hemolymph and measuring phenoloxidase activity in Tribolium castaneum
}

\author{
Hiroko Tabunoki ${ }^{1,2^{*}}$ (D) Neal T. Dittmer ${ }^{2}$, Maureen J. Gorman ${ }^{2}$ and Michael R. Kanost ${ }^{2}$
}

\begin{abstract}
Objective: Hemolymph plays many important roles in the physiology of an insect throughout its lifetime; however, for small-bodied insects, studies are lacking because of the difficulties encountered while collecting hemolymph. The objective of our study was to develop a method to collect hemolymph plasma from various stages of Tribolium castaneum and to evaluate phenoloxidase activity in the plasma samples. We first designed a procedure for easily and quickly collecting clear hemolymph plasma from T. castaneum.

Results: By using this method, we collected approximately $5 \mu$ plasma from 30 individuals at the larval, pupal or adult stages. And then, we studied the expression of phenoloxidase by performing western blot analysis of the plasma samples and found that phenoloxidase is present in hemolymph in each developmental stage. We also measured phenoloxidase activity in control plasma and plasma treated with Gram-positive bacteria, Micrococcus /uteus. Phenoloxidase activity was greater in some of the M. luteus-treated plasma samples compared with control samples. Thus, we developed a method to collect hemolymph plasma that is suitable for studies of phenoloxidase activity.
\end{abstract}

Keywords: Phenoloxidase, Melanization, Hemolymph, Small insects, Innate immune response, Melanin, Dopamine, Tribolium castaneum

\section{Introduction}

Insect hemolymph plays many important roles in the physiology of an insect, such as transporting nutrients and hormones to cells and tissues, maintaining the correct moisture ratio and optimal body temperature, maintaining body shape, and protecting the insect from pathogens [1]. In addition, some protein components in hemolymph plasma can differ between females and males throughout the developmental process and thus play specific roles in females and males. By investigating the biochemical components of hemolymph, the insect species can be better characterized. In the case of a honeybee, methods for collecting hemolymph were sought, and antennae method for hemolymph sampling (AMHS) is

\footnotetext{
${ }^{*}$ Correspondence: $\mathrm{h}$ tabuno@cc.tuat.ac.jp

${ }^{1}$ Department of Science of Biological Production, Graduate School of Agriculture, Tokyo University of Agriculture and Technology, 3-5-8 Saiwai-cho, Fuchu, Tokyo 183-8509, Japan

Full list of author information is available at the end of the article
}

propounded as the quickest and most reliable method for collecting hemolymph [2, 3]. However, for many insects the collection and analysis of hemolymph has been difficult because of their tiny body size relative to the honeybee, and more methods need to be developed to resolve this issue [4-6].

The red flour beetle (Tribolium castaneum) is a model insect of the order Coleoptera. The genome of this species is well characterized, and methods to induce systemic RNA interference (RNAi) are available [7-10]. We were interested in understanding the immune response system in T. castaneum hemolymph; however, T. castaneum is a small-size insect, and collection of enough hemolymph for biochemical assays has been difficult.

Phenoloxidase (PO) is one of the plasma proteins in hemolymph [11]. It is expressed as an inactive zymogen, and is activated through specific proteolytic cleavage in response to wounding or infection [12]. Activated phenoloxidase hydroxylates tyrosine and oxidizes DOPA, dopamine, and dopamine derivatives, and subsequent 
chemical reactions lead to the formation of melanin [9]. Melanin participates in wound healing and immunerelated melanization $[13,14]$. Because we are interested in melanization, one of the goals of this study was to develop a plasma collection process that is compatible with phenoloxidase activity assays.

Here, we report a new method to easily and quickly collect clear hemolymph plasma from larval, pupal, and adult $T$. castaneum. Importantly, this method is compatible with analyses of phenoloxidase activity.

\section{Main text \\ Methods}

Insects

The T. castaneum GA-1 strain was used in all experiments. The insects were reared on whole-wheat flour containing $5 \%$ brewer's yeast [15]. All insects were kept at $30{ }^{\circ} \mathrm{C}$ with a 16 -h light/8-h dark cycle. We used last instar larvae which were selected using No. 25 sieve (Fisher scientific Inc., Waltham, MA, USA), pupae (0-4-day-old pupa), and adults (0-7-day-old) in this study.

\section{Bioinformatics}

The signal peptide sequences were analyzed using the SignalP 4.1 [16], and the molecular weights for PPO I (GenBank AY884063) and PPO II (GenBank AY884064) were predicted using Genetyx 13.0 (Genetyx Co. Ltd., Tokyo, Japan).

\section{Hemolymph collection from T. castaneum}

Tribolium castaneum larvae, pupae, and adults were counted $(\mathrm{n}=30)$ and transferred to a small handmade sieve (Fig. 1a). The sieve was made by attaching a Nitex Nylon screen $630 \mu \mathrm{m}$ (Thermo Fisher Scientific, Inc., Waltham, MA, USA) to part of a $50 \mathrm{ml}$ plastic centrifuge tube (Corning Co. Ltd., One Riverfront Plaza, NY, USA). The larvae, pupae, and adults were washed with distilled water containing $0.1 \%$ Tween, then washed with
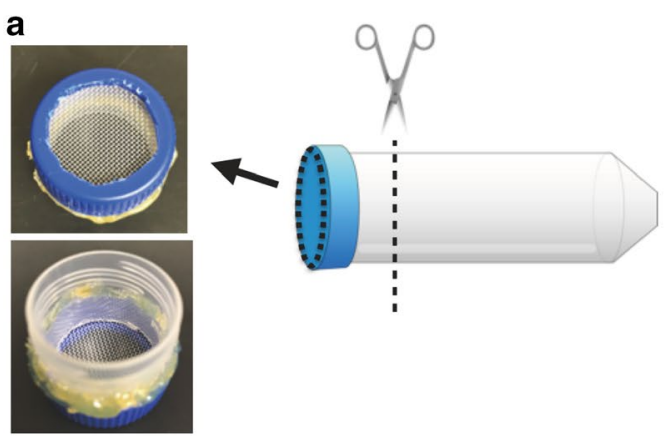

b

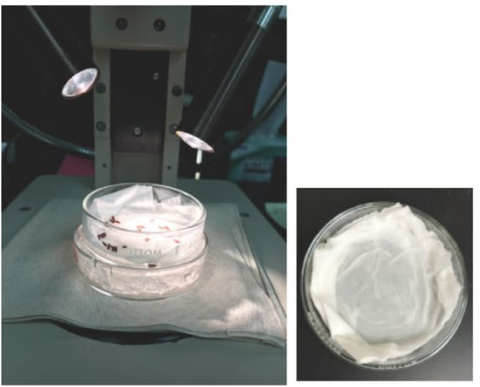

C

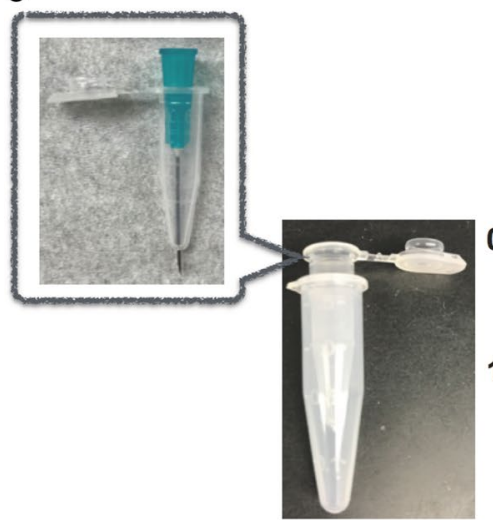

$0.5 \mathrm{ml}$ tube

$1.5 \mathrm{ml}$ tube

d

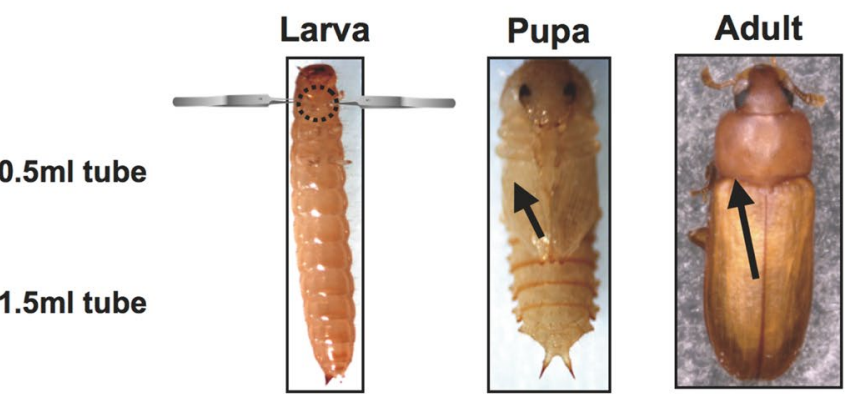

Fig. 1 Method for collecting hemolymph. a Creating a handmade sieve for washing T. castaneum. The $50 \mathrm{ml}$ tube drawing (http://g86.dbcls .jp/ togoriv/) is licensed under the HYPERLINK http://creativecommons.org/licenses/by/4.0/deed.ja. Creative commons display 4.0 license http:// creativecommons.org/licenses/by/4.0/deed.ja. b Set up of stereomicroscope. c Structure of handmade collection tube, which consisted of a $0.5 \mathrm{ml}$ tube with a hole at the bottom placed inside a $1.5 \mathrm{ml}$ tube. $\mathbf{d}$ Location of punctures created in the larvae, pupae, and adults. Larvae were pinched at the root of their legs using two forceps, and the legs were pulled in opposite directions. The black broken circle indicates the location for creating a small tear. The forceps drawings (http://g86.dbcls.jp/ togoriv/) are licensed under the HYPERLINK http://creativecommons.org/licen ses/by/4.0/deed.ja Creative commons display 4.0 license http://creativecommons.org/licenses/by/4.0/deed.ja. Pupae and adults were pierced with a needle at the points indicated by the black arrow. All protocols for collecting hemolymph from T. castaneum were performed on ice under a stereomicroscope 
tap water for $1 \mathrm{~min}$, and finally rinsed with $70 \%$ ethanol. Moisture was removed using a paper towel. The insects were then transferred to a glass dish containing a slightly moist and flat paper tissue on the bottom. The glass dish was kept on ice to anesthetize the insects (Fig. 1b). To prepare for collecting the hemolymph plasma, a hole was punctured in a 0.5 - $\mathrm{ml}$ tube using a 23 -gauge needle (Thermo Fisher Scientific, Inc.,) (Fig. 1c). To separate the males from the females, the sex of the pupal and adult insects was determined according to USDA Beetle Wrangling Tips, Sexing Tribolium [17] (Additional file 1: Figure S1).

To collect hemolymph from the larvae, pupae, and adults, T. castaneum individuals were handled as shown in Fig. 1d. The larvae were grasped at the base of their legs using two forceps (Fig. 1d, larva), and the legs were pulled in opposite directions to create a tear in the cuticle between them, with care to avoid damaging the gut. Pupae were pierced through the anterior region of an elytron (which in the pupa is on the ventral surface) using a 28 gauge needle (BD 1/2 cc U-100 Insulin Syringe \#329461) (Fig. 1d, pupa indicated by a black arrow). The adults were pierced on the dorsal side, between the pronotum and elytron (Fig. 1d, adult black arrow). The insects were then transferred to the ice-cold collection tubes (Fig. 1c). The collection tubes were centrifuged using the 5418 centrifuge (Eppendorf Inc. Co. Ltd. Hamburg, Germany) at $12,000 \times g$ for $10 \mathrm{~min}$ at $4{ }^{\circ} \mathrm{C}$, and the supernatant, which we refer to as plasma, was transferred to a new tube. For some experiments, this protocol was modified to prevent hemolymph melanization. For experiments measuring phenoloxidase activity, $20 \mu \mathrm{l}$ bleeding buffer (100 mM trisodium citrate, $17.8 \mathrm{mM}$ citric acid, $\mathrm{pH}$ 5.5) was placed at the bottom of the $1.5 \mathrm{ml}$ collection tubes. For samples used in immunodetection, $2 \mathrm{mg}$ phenylthiourea (Sigma-Aldrich, St. Louis, MO, USA) was added to the $1.5 \mathrm{ml}$ tube.

\section{Measurement of phenoloxidase activity}

The plasma collected using bleeding buffer was used to measure phenoloxidase activity. Two microliters of plasma were added to a $1.5-\mathrm{ml}$ tube containing $1 \mu \mathrm{l}$ filter-sterilized deionized water (control) or $1 \mu \mathrm{l}$ Micrococcus luteus $(1 \mu \mathrm{g} / \mu \mathrm{l}$ suspension, Sigma-Aldrich), and the tubes were incubated for $10 \mathrm{~min}$ at room temperature (RT). After incubation, these plasma samples were transferred to separate wells of a 96-well plate, after which $4 \mu \mathrm{l}$ of a $50 \mathrm{mM}$ dopamine hydrochloride solution in $1 \mathrm{mM}$ $\mathrm{HCl}$ (Sigma-Aldrich), and $93 \mu \mathrm{l}$ of $20 \mathrm{mM} \mathrm{3-(N-mor-}$ pholino)propanesulfonic acid (MOPS) (pH 7.0) containing $25 \mathrm{mM}$ calcium chloride (Sigma-Aldrich) were added to each well. The absorbance at $470 \mathrm{~nm}$ was monitored once/minute for $1 \mathrm{~h}$ at $30^{\circ} \mathrm{C}$ using a microplate spectrophotometer (Synergy HT, BioTek co. ltd., Winooski, VT USA). The reaction curves started with a lag phase followed by a linear phase. Linear regression was used to calculate the changes in absorbance per minute during the linear phase of each reaction. One unit of phenoloxidase activity was defined as $\Delta \mathrm{A}_{470}=0.001 / \mathrm{min}$ [18]. The assay was performed in triplicate. Statistical significance was determined by two-tailed Student's t-test using Excel (Microsoft, Redmond, WA, USA). p values of $<0.05$ were considered to be significant.

\section{Western blotting}

Plasma samples $(0.5 \mu \mathrm{l})$ containing phenylthiourea were heated in SDS-PAGE sample buffer and then separated by electrophoresis on $4-12 \%$ BisTris gels with MOPS running buffer (Thermo Fisher Scientific, Inc.) under reducing conditions according to the manufacturer's instructions. The proteins were then transferred to nitrocellulose membranes (Bio-Rad Laboratories, Inc., Hercules, CA, USA) using the method described by Towbin et al. [19]. The membranes were incubated in blocking buffer composed of 3\% milk and Tris-buffered saline (TBS) at pH 7.4, including 0.1\% Tween 20 (TBS-T) for $1 \mathrm{~h}$ at RT, incubated in rabbit anti-phenoloxidase polyclonal antiserum (KSU37) as the primary antibody (diluted 1:2000) in blocking buffer for $1 \mathrm{~h}$, and washed three times with TBS-T for $5 \mathrm{~min}$. The polyclonal antiserum KSU37 used for immunoblot analysis detects both the PPOI and PPOII subunits of Manduda sexta prophenoloxidase [20]. The washed membranes were incubated with goat anti-rabbit immunoglobulin $(\mathrm{Ig}) \mathrm{G}(\mathrm{H}+\mathrm{L})$ alkaline phosphatase (AP) conjugate 1:3000 (Bio-Rad Laboratories, Inc.) in blocking buffer for $1 \mathrm{~h}$ at RT and then washed twice with TBS-T for $5 \mathrm{~min}$ and with TBS for $5 \mathrm{~min}$. The membranes were developed using an AP Conjugate Substrate Kit (Bio-Rad Laboratories, Inc.) for $6 \mathrm{~min}$.

\section{Results and discussion}

In this study, we developed methods for easily and quickly collecting clear plasma from T. castaneum and assessed the presence and activity of phenoloxidase in the plasma. First, we collected approximately $5 \mu \mathrm{l}$ of hemolymph from 30 individuals at each developmental stage (Additional file 1: Table S1).

Tribolium castaneum expresses two phenoloxidase genes: PPOI and PPOII [21]. PPOI has a predicted molecular weight of 79,099 $\mathrm{Da}$ and PPOII has a predicted molecular weight of 79,338 $\mathrm{Da}$. We examined the utility of available antisera against $M$. sexta phenoloxidase to identify phenoloxidase in T. castaneum plasma. M. sexta anti-phenoloxidase antibody reacted with one or two 

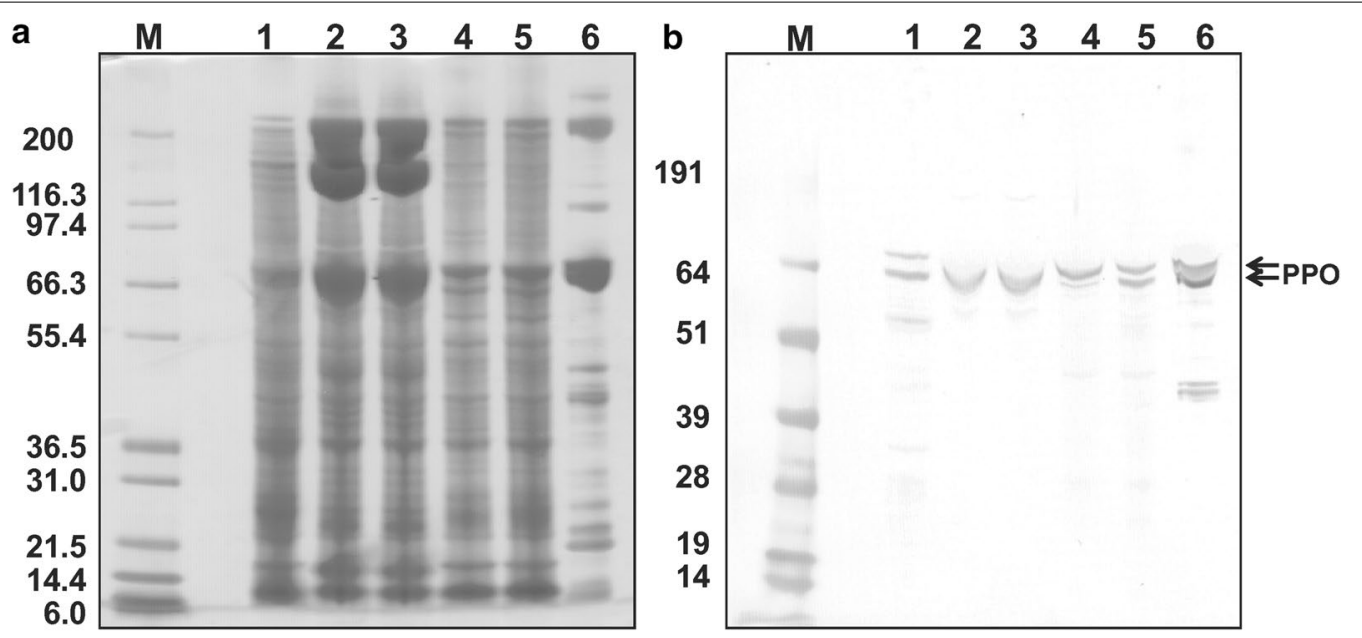

Fig. 2 Dectection of phenoloxidase in larvae, pupae and adults. Plasma protein samples ( $0.5 \mu \mathrm{l})$ from T. castaneum larva, lane 1; male pupa, lane 2; female pupa, lane 3; male adult, lane 4; and female adult, lane 5; M. sexta larval plasma, lane 6 (as a positive control) were separated by SDS-PAGE, transferred to nitrocellulose, and probed with anti-M. sexta phenoloxidase antibody. a Coomassie blue staining of the separated proteins. b Immunoblotting. Lane M represents molecular weight markers. Arrows indicate bands of the expected size for prophenoloxidase detected by prophenoloxidase antiserum

polypeptides of approximately $80 \mathrm{kDa}$ in all T. castaneum plasma samples (Fig. 2b). Because phenoloxidase bands of the expected size were detected, we conclude that the plasma samples were not significantly contaminated with gut contents (which contain proteolytic enzymes) (Fig. 2a). Also, we found that collected hemolymph plasma may include some cellular proteins because our samples contained many more protein bands than plasma from Tenebrio molitor, which is a larger Coleopteran insect than T. castaneum [22].

It has been reported that neither PPO mRNA was expressed from the late pupa stage to the early adult stage [21]. However, phenoloxidase was found in adult plasma in this study. These results suggest that $T$. castaneum phenoloxidase is a stable protein, persisting from the pupal stage into the adult stage.

We attempted to measure phenoloxidase activity in $T$. castaneum plasma by monitoring the oxidation of the substrate dopamine according to a previously described phenoloxidase assay [18]. However, this method was not suitable for T. castaneum plasma because of rapid hemolymph melanization that occurred in the larval and adult samples before the assay could be performed.

To circumvent this problem, we tested several bleeding and assay buffers to determine which combinations would best suppress oxidation of endogenous catechols during the collection process but give reliable results during the activity assay; these buffer combinations are shown in Additional file 1: Table S2. Buffer combinations 1 to 3 were not suitable for measuring phenoloxidase activity as the change in absorbance did not progress linearly with time (Additional file 1: Figures S2, S3, S4, Table S3).

The assay was slightly improved using buffer combination 4 (Additional file 1: Figure S5, Table S3), as this prevented premature melanization in the larval plasma but not the adult plasma. Previous reports for Tenebrio molitor, Alphitobius diaperinus, and Hermetia illucens indicated that oxidation of endogenous dopamine was inhibited using a bleeding buffer at $\mathrm{pH} 4.0$; however, phenoloxidase activity was highest at approximately $\mathrm{pH} 6.0$ in these species [23]. Thus, we assessed additional buffer combinations according to our previous findings. Buffer combination 5 completely blocked early plasma melanization, however phenoloxidase activity was very low in all samples (Additional file 1: Figure S6, Table S3). Finally, we found that buffer combination 6 , with collecting hemolymph into citrate buffer at $\mathrm{pH} 5.5$, was suitable for minimizing melanization during hemolymph collection while still allowing for sufficient dopamine oxidation for measuring phenoloxidase activity (Additional file 1: Figure S7, Fig. 3).

For some studies of insect immunity, phenoloxidase in hemolymph or plasma is intentionally activated by the addition of an immune elicitor. To verify that the plasma collection method we developed is suitable for these kinds of studies, we observed whether phenoloxidase activity was higher after incubating the plasma with Gram-positive bacteria, M. luteus. For these experiments, we used buffer combination 6 . We found that 


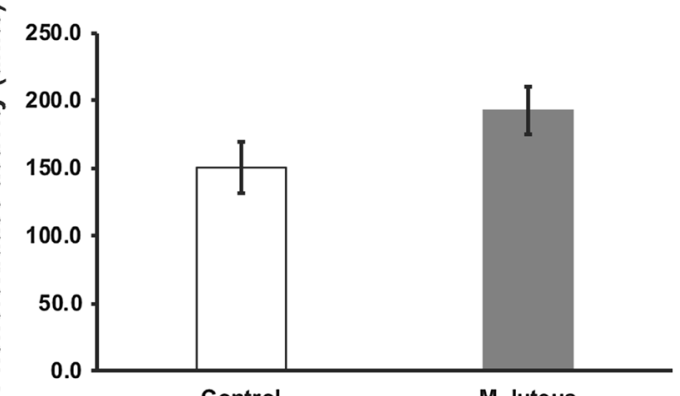

250.0

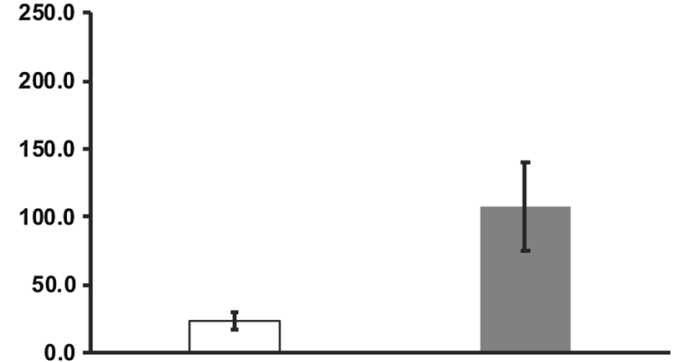

Control

M. luteus

250.0

200.0

150.0

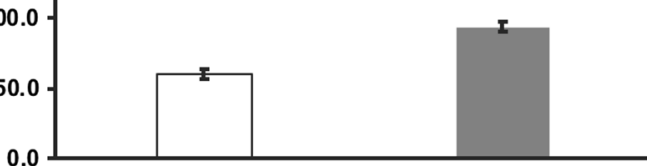

M. luteus

Fig. 3 Phenoloxidase activity of plasma samples from T. castaneum. Control, no added bacteria; M. luteus indicates plasma samples treated with M. luteus prior to PO assay. $\mathbf{a}$ Larva $(p=0.183)$, $\mathbf{b}$ male pupa $(p=0.065)$, $\mathbf{c}$ female pupa $(p=0.003)$, $\mathbf{d}$ male adult $(p=0.008)$, and $\mathbf{e}$ female adult $(p=0.007)$. Error bars indicate standard errors $(n=3)$

phenoloxidase activity increased after treatment with $M$. luteus in plasma samples from female pupae and male and female adults, but the increase observed in plasma from larvae and male pupae was not statistically significant (Fig. 3). It is possible that the phenoloxidase in those samples was already partially activated by the collection process.

\section{Conclusion}

We developed an easy method to collect hemolymph plasma from T. castaneum. Our methods allow for the conducting of experiments which have previously been challenging due to the difficulty in collecting sufficient amounts of hemolymph from this insect. Also, we were d
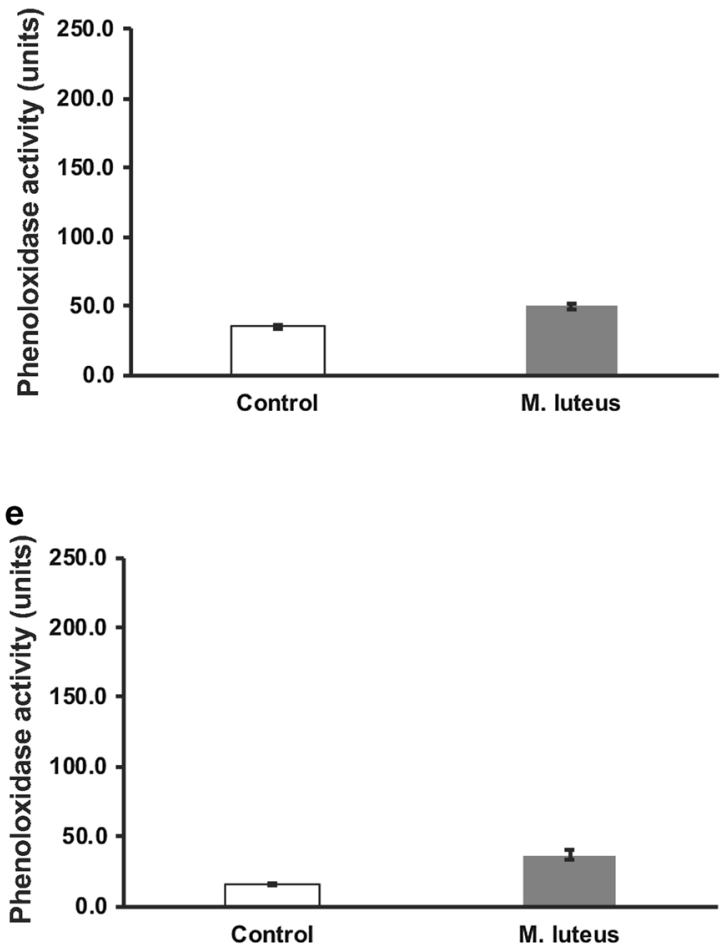

able to clarify that prophenoloxidase protein is a stable protein, persisting from the pupal stage into the adult stage of the T. castaneum hemolymph. This new method could be helpful for various analyses of $T$. castaneum hemolymph, for example, biochemical assays, other enzyme assays, proteome analysis, metabolomic analysis, and tracking of labeled substances or proteins in the hemolymph in T. castaneum.

\section{Limitations}

This method may not be suitable for studying hemocytes as they are removed and possibly damaged during the centrifugation step. This method may also damage other cells whose contents may then contaminate the plasma sample. 


\section{Additional file}

Additional file 1: Figure S1. Identifying the sex of pupal and adult $T$. castaneum. The structure of the genital papillae is markedly different between female and male pupae. The pupae were sexed by examining the structure of the genital papillae (on the last abdominal segment), which are markedly different between female and male pupae (indicated with black arrow). For adults, sex was determined by the presence (male) or absence (female) of a patch of bristles ("sex patch"; indicated by white arrow) on the femur of the prothoracic legs. Figure S2. Observation of the reaction curve in experimental condition 1. The absorbance (A470) was monitored, and calculated as $\mathrm{mOD} . \mathrm{D} / \mathrm{min}(\triangle \mathrm{A} 470=0.001 / \mathrm{min})$, and plotted on the graph. Control, no added bacteria; $M$. luteus indicates plasma samples treated with M. luteus prior to PO assay. A; larva control, B; larva M. luteus, C; Adult control, D; Adult M. luteus. Figure S3. Observation of the reaction curve in Experimental condition 2. The absorbance (A470) was monitored, and calculated as $\mathrm{mO}$.D. $/ \mathrm{min}(\triangle \mathrm{A} 470=0.001 / \mathrm{min})$, and plotted on the graph. Control, no added bacteria; $M$. Iuteus indicates plasma samples treated with M. luteus prior to PO assay. A; larva control, B; larva M. luteus, C; Adult control, D; Adult M. Iuteus. Figure S4. Observation of the reaction curve in Experimental condition 3. The absorbance (A470) was monitored, and calculated as $\mathrm{mO}$.D. $/ \mathrm{min}(\triangle \mathrm{A} 470=0.001 / \mathrm{min})$, and plotted on the graph. Control, no added bacteria; M. Iuteus indicates plasma samples treated with M. luteus prior to PO assay. A; larva control, B; larva $M$. luteus, C; Adult control, D; Adult M. luteus. Figure S5. Observation of the reaction curve in Experimental condition 4. The absorbance (A470) was monitored, and calculated as mO.D. $/ \mathrm{min}(\triangle \mathrm{A} 470=0.001 / \mathrm{min})$, and plotted on the graph. Control, no added bacteria; M. luteus indicates plasma samples treated with $M$. luteus prior to PO assay. A; larva control, B; larva M. luteus, C; Adult control, D; Adult M. luteus. Figure S6. Observation of the reaction curve in Experimental condition 5. The absorbance (A470) was monitored, and calculated as $\mathrm{mO}$.D. $/ \mathrm{min}(\triangle \mathrm{A} 470=0.001 / \mathrm{min})$, and plotted on the graph. Control, no added bacteria; $M$. Iuteus indicates plasma samples treated with M. luteus prior to PO assay. A; larva control, B; larva M. Iuteus, C; Adult control, D; Adult M. Iuteus. Figure S7. Observation of the reaction curve in Experimental condition 6. The absorbance $\left(A_{470}\right)$ was monitored, and calculated as $\mathrm{mO}$.D. $/ \mathrm{min}(\triangle \mathrm{A} 470=0.001 / \mathrm{min})$, and plotted on the graph. Control, no added bacteria; $M$. luteus indicates plasma samples treated with M. luteus prior to PO assay. A; larva control, B; larva M. luteus, C; Pupa-male control, D; Pupa-male M. luteus, E; Pupa-female control, F; Pupa-female M. luteus, G; Adult-male control, H; Adult-male M. Iuteus, l; Adult-female control, J; Adult-female M. luteus. Table S1. Plasma collected from different developmental stages $(n=30)$. Table S2. Assay buffer combinations tested for measuring phenoloxidase (PO) activity. Table S3. Phenoloxidase activity in experimental combination 1 to 5 .

\section{Abbreviations}

AMHS: antennae method for hemolymph sampling; RNAi: RNA interference; PO: phenoloxidase.

\section{Authors' contributions}

Conceived and designed the experiments HT, MJG, NTD and MRK. Contributed reagents/materials/analysis tools MJG, NTD and MRK. Performed the experiments HT. Analyzed the data HT, MJG, NTD and MRK. Contributed to the writing of the paper HT, MJG, NTD and MRK. All authors ensure that this is the case. All authors read and approved the final manuscript.

\section{Author details}

1 Department of Science of Biological Production, Graduate School of Agriculture, Tokyo University of Agriculture and Technology, 3-5-8 Saiwai-cho, Fuchu, Tokyo 183-8509, Japan. ${ }^{2}$ Department of Biochemistry and Molecular Biophysics, Kansas State University, 141 Chalmers Hall, Manhattan, KS 66506-3702, USA.

\section{Acknowledgements}

We thank Dr. Karl Kramer for advice about hemolymph plasma proteins in T. castaneum, and Ms. Lisa Brummett for helping with the Western blotting experiment. This work was supported by Science and Technology of Japan and Overseas travel assistance program supported by TUAT president discretionary expenses to $\mathrm{HT}$.

\section{Competing interests}

The authors declare that they have no competing interests.

\section{Availability of data and materials}

All experimental data in this study were included in this article. Additional figures and tables are available in this article.

\section{Consent for publication}

Not applicable.

\section{Ethics approval and consent to participate}

Not applicable.

\section{Funding}

This work was supported by Funds by the Japan Society for the Promotion of Science (JSPS) Grant-in-Aid for Scientific Research B (18H02212) and by National Institute of General Medical Sciences Grant R37 GM41247. The funders did not have a role in the design of the study and collection, analysis, and interpretation of data and in writing the manuscript.

\section{Publisher's Note}

Springer Nature remains neutral with regard to jurisdictional claims in published maps and institutional affiliations.

Received: 29 August 2018 Accepted: 31 December 2018

Published online: 07 January 2019

\section{References}

1. Nation JL. Insect physiology and biochemistry. 3rd ed. Florida: CRC Press; 2016. p. 420-4.

2. Łoś A, Strachecka A. Fast and cost-effective biochemical spectrophotometric analysis of solution of insect "blood" and body surface elution. Sensors (Basel). 2018;18(5):1494.

3. Borsuk G, Ptaszyńska AA, Olszewski K, Domaciuk M, Krutmuang P, Paleolog J. A new method for quick and easy hemolymph collection from apidae adults. PLoS ONE. 2017;12(1):e0170487.

4. Joop G, Roth O, Schmid-Hempel P, Kurtz J. Experimental evolution of external immune defences in the red flour beetle. J Evol Biol. 2014;27(8):1562-71.

5. Yokoi K, Hayakawa Y, Kato D, Minakuchi C, Tanaka T, Ochiai M, et al. Prophenoloxidase genes and antimicrobial host defense of the model beetle, Tribolium castaneum. J Invertebr Pathol. 2015;132:190-200.

6. Rafaluk-Mohr C, Wagner S, Joop G. Cryptic changes in immune response and fitness in Tribolium castaneum as a consequence of coevolution with Beauveria bassiana. J Invertebr Pathol. 2018;152:1-7.

7. Kim HS, Murphy T, Xia J, Caragea D, Park Y, Beeman RW, et al. BeetleBase in 2010: revisions to provide comprehensive genomic information for Tribolium castaneum. Nucleic Acids Res. 2010;38:D437-42.

8. Tribolium Genome Sequencing Consortium. The genome of the model beetle and pest Tribolium castaneum. Nature. 2008:452:949-55.

9. Knorr E, Bingsohn L, Kanost MR, Vilcinskas A. Tribolium castaneum as a model for high-throughput RNAi screening. Adv Biochem Eng Biotechnol. 2013;136:163-78.

10. Schmitt-Engel C, Schultheis D, Schwirz J, Ströhlein N, Troelenberg N, Majumdar U, et al. The iBeetle large-scale RNAi screen reveals gene functions for insect development and physiology. Nat Commun. 2015;6:7822.

11. Kanost MR, Gorman MJ. Phenoloxidases in insect immunity. In: Beckage $\mathrm{N}$, editor. Insect immunology. San Diego: Academic Press/Elsevier; 2008. p. 69-86.

12. Cerenius L, Soderhall K. The prophenoloxidase-activating system in invertebrates. Immunol Rev. 2004;198:116-26.

13. Christensen BM, Li J, Chen CC, Nappi AJ. Melanization immune responses in mosquito vectors. Trends Parasitol. 2005;21:192-9. 
14. Nappi AJ, Christensen BM. Melanogenesis and associated cytotoxic reactions: applications to insect innate immunity. Insect Biochem Mol Biol. 2005;35:443-59.

15. Lasley EL, et al. Tribolium information bulletin. In: William HM, editor. Agricultural research institute. New York: Chazy; 1960. p. 3-13.

16. The SignalP 4.1 server: http://www.cbs.dtu.dk/services/SignalP/. Accessed 29 Jun 2018.

17. USDA beetle wrangling tips, sexing Tribolium: https://www.ars.usda.gov/ plains-area/mhk/cgahr/spieru/docs/tribolium-stock-maintenance/\#sexin g. Accessed 15 Jun 2018.

18. Gorman MJ, An C, Kanost MR. Characterization of tyrosine hydroxylase from Manduca sexta. Insect Biochem Mol Biol. 2007;37:1327-37.

19. Towbin H, Staehelin T, Gordon J. Electrophoretic transfer of proteins from polyacrylamide gels to nitrocellulose sheets: procedure and some applications. Proc Natl Acad Sci USA. 1979;76:4350-4
20. Jiang H, Wang Y, Ma C, Kanost MR. Subunit composition of pro-phenol oxidase from Manduca sexta: molecular cloning of subunit ProPO-P1. Insect Biochem Mol Biol. 1997:27:835-50.

21. Arakane Y, Muthukrishnan S, Beeman RW, Kanost MR, Kramer KJ. Laccase 2 is the phenoloxidase gene required for beetle cuticle tanning. Proc Natl Acad Sci USA. 2005;102:11337-42.

22. Zhang R, Cho HY, Kim HS, Ma YG, Osaki T, Kawabata S, et al. Characterization and properties of a 1,3-beta-D-glucan pattern recognition protein of Tenebrio molitor larvae that is specifically degraded by serine protease during prophenoloxidase activation. J Biol Chem. 2003;278(43):42072-9.

23. Janssen RH, Lakemond CMM, Fogliano V, Renzone G, Scaloni A, Vincken JP. Involvement of phenoloxidase in browning during grinding of Tenebrio molitor larvae. PLoS ONE. 2017;12(12):e0189685.
Ready to submit your research? Choose BMC and benefit from:

- fast, convenient online submission

- thorough peer review by experienced researchers in your field

- rapid publication on acceptance

- support for research data, including large and complex data types

- gold Open Access which fosters wider collaboration and increased citations

- maximum visibility for your research: over $100 \mathrm{M}$ website views per year

At BMC, research is always in progress.

Learn more biomedcentral.com/submissions 